\title{
Perkutaner Verschluss des linken Vorhofohres senkt die Schlaganfallrate
}

Fragestellung: Kann ein perkutaner Verschluss des linken Vorhofohres bei Patienten mit Vorhofflimmern im Vergleich zu einer oralen Antikoagulation mit einem Vitamin-K-Antagonisten wie Warfarin Schlaganfälle verhindern?

Hintergrund: Patienten mit Vorhofflimmern haben ein deutlich erhöhtes Schlaganfallrisiko. Dies kann durch eine orale Antikoagulation beispielsweise mit Vitamin-K-Antagonisten wie Warfarin signifikant reduziert werden. Seit längerem ist aus echokardiografischen Studien bekannt, dass die wesentliche Quelle für die Bildung von Thromben das linke Vorhofohr ist. Aus diesem Grund wurden in den letzten Jahren Verschlusssysteme entwickelt, mit denen perkutan das linke Vorhofohr verschlossen werden kann. Eines dieser Devices wurde in der PROTECT-AF-Studie untersucht $[1,2]$. Nach einer durchschnittlich zweijährigen Beobachtungszeit gab es keinen signifikanten Unterschied zwischen dem perkutanem Verschluss des linken Vorhofsohres und Warfarin bezogen auf Schlaganfälle, systemische Embolien und Todesfälle. Mit dieser Studie wird eine weitere Analyse mit einer deutlich längeren Beobachtungszeit vorgelegt.

Reddy VY, Sievert H, Halperin J et al; PROTECT AF Steering Committee and Inves-tigators. Percutaneous left atrial appendage closure vs warfarin for atrial fibrillation: a randomized clinical trial. JAMA 2014; 312: $1988-98$
Patienten und Methodik: PROTECT AF war eine multizentrische randomisierte offene Studie, die an 59 Krankenhäusern durchgeführt wurde. Eingeschlossen wurden 707 Patienten mit nicht valvulärem Vorhofflimmern und mindestens einem Schlaganfallrisikofaktor. Die Patienten wurden im Verhältnis 2:1 randomisiert und erhielten entweder zwischen Februar 2005 und Juni 2008 einen perkutanen Verschluss des linken Vorhofohres mit dem WATCHMAN-Verschlusssystem der Firma Boston Scientific oder sie wurden oral antikoaguliert. Der Endpunkt der Studie war Schlaganfall, systemische Embolien und kardiovaskuläre sowie unerklärte Todesfälle. Bei allen Patienten erfolgte über 45 Tage eine Behandlung mit Warfarin und Acetylsalicylsäure.

Ergebnisse: Nach durchschnittlich 3,8 Jahren Beobachtungszeit und 2.621 Patientenjahren ergaben sich 39 primäre Ereignisse bei den 463 Patienten in der Verschlussgruppe (8,4\%). In der Antikoagulationsgruppe zeigten sich 34 Ereignisse bei 244 Patienten (13,9\%). Dieser Unterschied war statistisch signifikant.

Signifikante Unterschiede ergaben sich auch für die Gesamtsterblichkeit und die kardiovaskuläre Sterblichkeit. Erwartungsgemäß traten schwerwiegende Perikardergüsse nur in der Interventionsgruppe auf. Dort war die Ereignisrate 4,8\%. Alle anderen Endpunkte inklusive schwerwiegender Blutungskomplikationen waren in beiden Gruppen gleich häufig.

Schlussfolgerungen: Im Langzeitverlauf zeigt sich bei Patienten mit nicht valvulärem Vorhofflimmern eine geringe, aber statistisch signifikante Überlegenheit für den perkutanen Verschluss des linken Vorhofohres, verglichen mit einer oralen Antikoagulation mit Warfarin. Allerdings kommt es bei dem interventionellen Eingriff relativ häufig zu schwerwiegenden Perikardergüssen und Blutungen.

\section{- Kommentar von Hans-Christoph Diener, Essen}

\section{Gelten die Ergebnisse auch für Patienten mit erhöhtem Blutungsrisiko?}

Die PROTECT-AF-Studie war für den ursprünglichen Endpunkt und die ursprüngliche Beobachtungsdauer negativ. Hier handelt es sich jetzt um eine Langzeitauswertung, die einen kleinen signifikanten Unterschied zugunsten des perkutanen Verschlusses des linken Vorhofohres zeigt. Die Studie beantwortet allerdings nicht eine sehr kritische Frage, nämlich ob dieser Eingriff für Patienten infrage kommt, die entweder eine zerebrale Blutung unter Antikoagulation erlitten haben oder bei denen generell ein hohes Blutungsrisiko besteht, wenn sie antikoaguliert werden. Da die Patienten nach der Implantation des Devices vorübergehend antikoaguliert werden müssen, kommen sie leider für diesen Eingriff nicht in Betracht. Wünschenswert wäre allerdings eine Studie an Patienten mit erhöhtem Blutungsrisiko, bei denen es vertretbar wäre, über einen Zeitraum von 45 Tagen zu antikoagulieren.
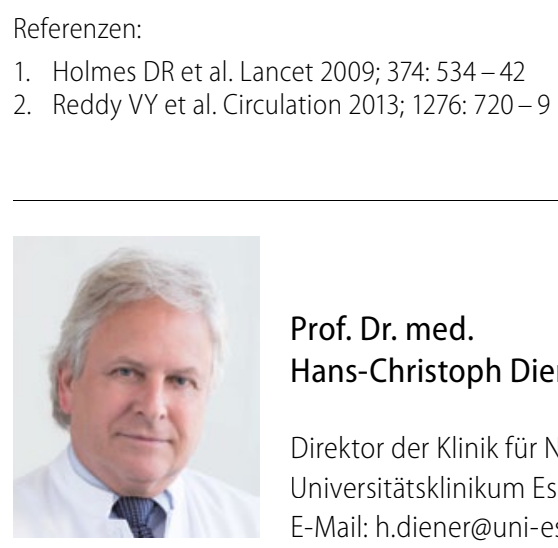

Prof. Dr. med. Hans-Christoph Diener, Essen

Direktor der Klinik für Neurologie, Universitätsklinikum Essen E-Mail:h.diener@uni-essen.de 